\title{
Vasculitis Associated with Chronic Lymphocytic Leukemia
}

\section{Kunnath $\mathrm{TP}^{1}$, Rong $\mathrm{R}^{2}$ and Ambrus JL Jr ${ }^{* 1}$}

${ }^{1}$ Department of Medicine, SUNY at Buffalo School of Medicine, Buffalo, NY, USA

${ }^{2}$ Department of Pathology, SUNY at Buffalo School of Medicine, Buffalo, NY, USA

*Corresponding author: Ambrus JL Jr, MD., Department of Medicine, SUNY at Buffalo School of Medicine, Buffalo, NY, USA 14203, E-mail: jambrus@buffalo.edu

Citation: Kunnath TP, Rong R, Ambrus JL (2015) Vasculitis Associated with Chronic Lymphocytic Leukemia.

J Case Rep Stud 3(5): 505. doi: 10.15744/2348-9820.3.505

Received Date: July 20, 2015 Accepted Date: October 12, 2015 Published Date: October 14, 2015

\section{Abstract}

A patient is present who has chronic lymphocytic leukemia (CLL) and developed a vasculitis involving the skin and large vessels along with immune complex mediated glomerulosclerosis. Both vasculitis and immune complex nephritis are rare manifestations of CLL.

Keywords: Vasculitis; CLL

Abbreviations: CLL: Chronic Lymphocytic Leukemia; GN: Glomerulonephritis

\section{Introduction}

Chronic Lymphocytic leukemia is associated with a variety of autoimmune phenomenon, especially autoimmune cytopenias [1]. The incidence of vasculitis in CLL is very low, approximately $0.1-2 \%$ and is generally a leukocytoclastic vasculitis restricted to the skin $[2,3]$. We report here a rare case of systemic vasculitis associated with CLL.

\section{Case presentation}

The patient is a 60 year-old Caucasian male with multiple comorbidities including coronary artery disease, paroxysmal atrial fibrillation and stage four B cell - CLL, diagnosed in May 2013. He had undergone 3 cycles of chemotherapy with Rituximab and Bendamustine. He presented with a maculo-papular rash 4 weeks after his last chemotherapy cycle that initially started in his lower extremities and spread to his entire body. His CBC with 3.2\% lymphocytes did not suggest a flare of disease. He had stage 3 chronic kidney disease with a baseline creatinine of 1.2 that was attributed to renal artery stenosis. At the time of his presentation, his creatinine rose from $1.2 \mathrm{mg} / \mathrm{dL}$ to $5.12 \mathrm{mg} / \mathrm{dL}$. He had low C3 $(65 \mathrm{mg} / \mathrm{dl})$ and red cells in the urine. He had a positive ANA (titer1: 160). He had negative cryoglobulins and normal SPEP and UPEP. ASO Antibody titer was negative as were serologies for HSV, CMV and parvovirus. He underwent kidney and skin biopsies. The immunofluorescence studies of the kidney biopsy showed IgG - 2+, IgA -2+, IgM- 3+ and C3-2+ deposition. The light microscopy showed focal segmental glomerulosclerosis. Based on the IF and EM results, this is likely a secondary form of focal segmental glomerulosclerosis associated with immune complex deposition (Figure 1A). Electron microscopy showed immune complex deposition in the sub epithelial spaces of the basement membrane (Figure 1B). The skin biopsy showed necrosis of the epidermis with neutrophilic infiltration and leukocytoclastic vasculitis (Figure 1C). A CT angiogram of the abdomen showed marked irregularity in contour of the mucosal surface of the lumen of the abdominal aorta consistent with aortitis (Figure 2). Treatment with systemic corticosteroids led to resolution of his skin lesions and improvement of his renal function back to baseline. He subsequently received a fourth cycle of chemotherapy. He later had progression of his CLL.

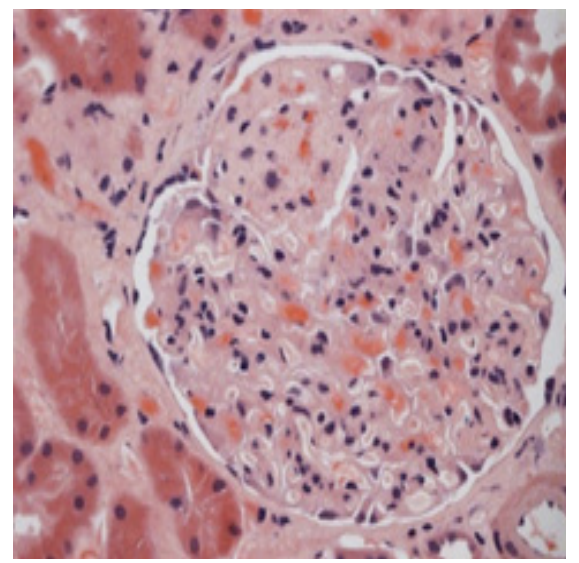

Figure 1A: Light microscopy of the kidney biopsy showing focal segmental glomerulosclerosis 


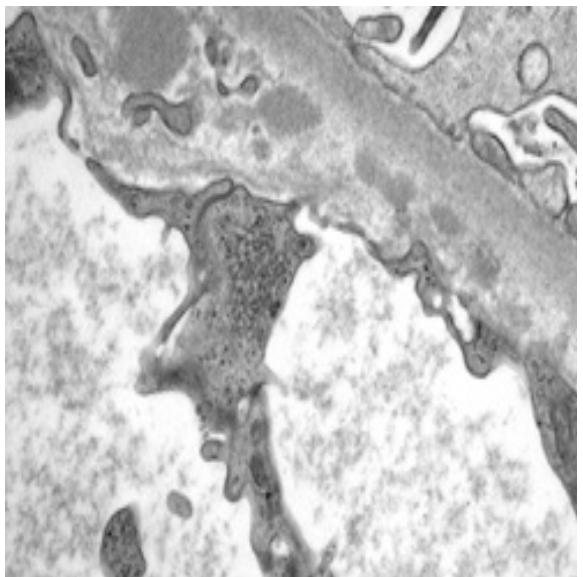

Figure 1B: Electron microgram of the kidney showing immune complex deposition in the sub epithelial spaces of the basement membrane

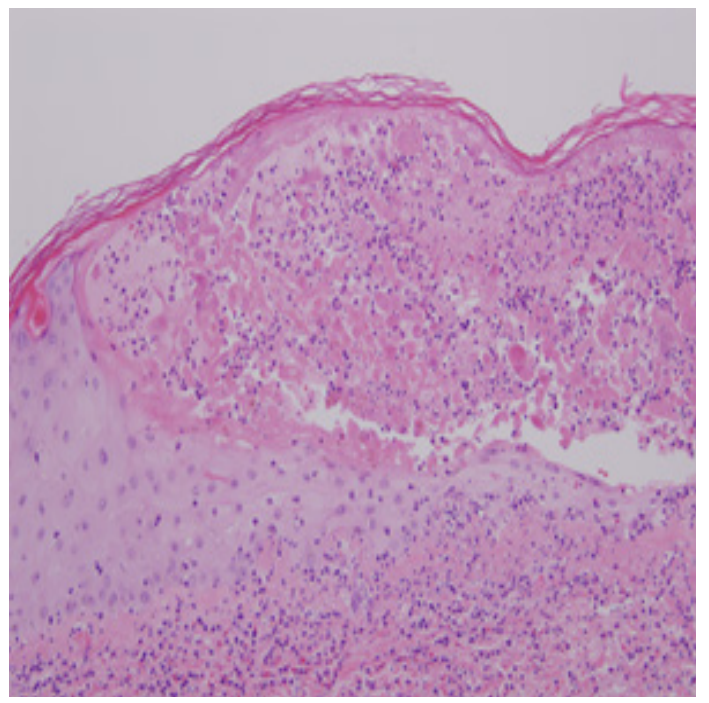

Figure 1C: Hematoxylin and Eosin Stain of the skin biopsy

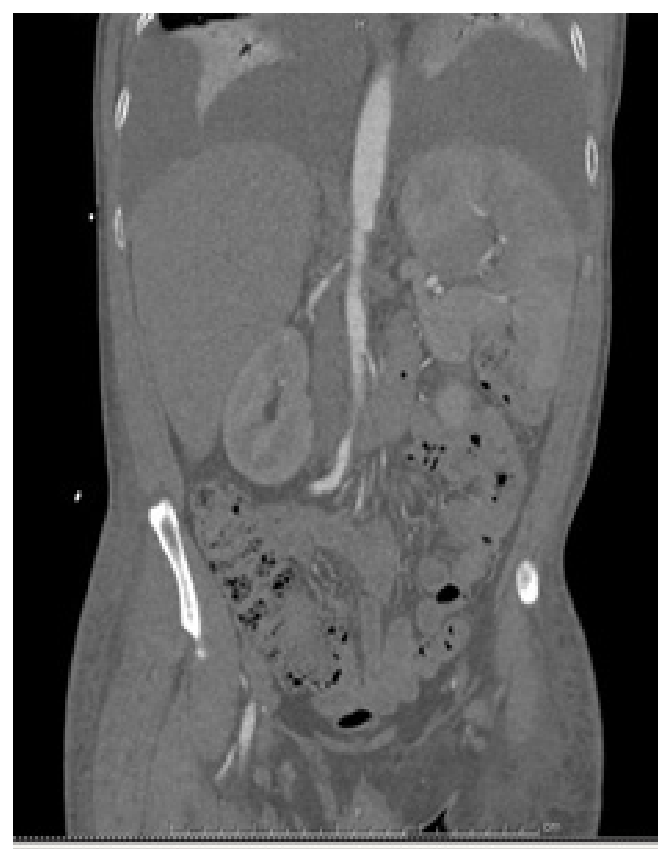

Figure 2: CTA of the abdomen showing aortic dilatation 


\section{Discussion}

Various malignancies have been associated with vasculitis. Most commonly this is a leukocytoclastic vasculitis [3]. However, vasculitides associated with malignancy have occasional presented with clinical pictures consistent with polyarteritis nodosa, Churg-Strauss syndrome, microscopic polyangiitis, Wegener's granulomatosis and Henoch-Schönlein purpura [4]. Vasculitis with malignancy may occur because of multiple mechanisms including formation of immune complexes to neoplastic antigens, polyclonal activation of B-lymphocytes, aggregation of monoclonal immunoglobulins, formation of antibodies directed towards endothelial antigens, infiltration of the vascular wall by the malignancy, secondary infections and/ or immune reactivity to anticancer drugs [5-7]. In our patient there was evidence for vasculitis involving the skin and the large blood vessels. The kidney showed immune complex deposition, but the renal lesion was predominantly focal sclerosing glomerulonephritis with immune complexes in the basement membrane. Membranous nephropathy is a well-known glomerular disease associated with lymphoproliferative disorders [8]. The link between CLL and glomerulonephritis is generally an abnormal immunoglobulin produced by a malignant $\mathrm{B}$ cell clone that acts either as a cryoglobulin or an aggregate that gets trapped in the basement membrane.

In the case presented, the patient responded nicely to systemic corticosteroids.

\section{Conclusion}

Systemic vasculitis in CLL is an extremely rare entity. We present an unusual case of systemic vasculitis with skin and large vessel involvement along with membranous glomerulonephritis.

\section{Consent}

"Written informed consent was obtained from the patient for publication of this case report and accompanying images.

\section{Authors' contributions}

TPK and JLA both participated in the care of the patient and the preparation of this manuscript. RR reviewed the pathology and contributed to the preparation of the manuscript.

\section{Acknowledgement}

The authors would like to thank Dr. Jessica Sugianto for review of the skin biopsies.

\section{References}

1. Zent CS, Kay NE (2010) Autoimmune complications in chronic lymphocytic leukaemia (CLL). Best Pract Res Clin Haematol 23: 47-59.

2. Pavlidis NA, Klouvas G, Tsokos M, Bai M, Moutsopoulos HM (1995) Cutaneous lymphocytic vasculopathy in lymphoproliferative disorders--a paraneoplastic lymphocytic vasculitis of the skin. Leuk Lymphoma 16: 477-82.

3. Yadav BS, Sharma SC, Kapoor RK (2006) Paraneoplastic leukocytoclastic vasculitis in chronic lymphoid leukemia. J Cancer Res Ther 2: 206-8.

4. Fain O, Hamidou M, Cacoub P, Godeau B, Wechsler B, et al. (2007) Vasculitides associated with malignancies: analysis of sixty patients. Arthritis Rheum 57: 1473-80.

5. Greer JM, Longley S, Edwards NL, Elfenbein GJ, Panush RS (1992) Vasculitis associated with malignancy. Experience with 13 patients and literature review. Medicine 67: 220-30.

6. Mertz LE, Conn DL (1992) Vasculitis associated with malignancy. Curr Opin Rheumatol 4: 39-46.

7. Chen KR, Carlson JA (2008) Clinical approach to cutaneous vasculitis. Am J Clin Dermatol 9: 71-92.

8. Moulin B, Ronco PM, Mougenot B, Francois A, Fillastre JP, et al. (1922) Glomerulonephritis in chronic lymphocytic leukemia and related B-cell lymphomas. Kidney Int 42: 127-35.

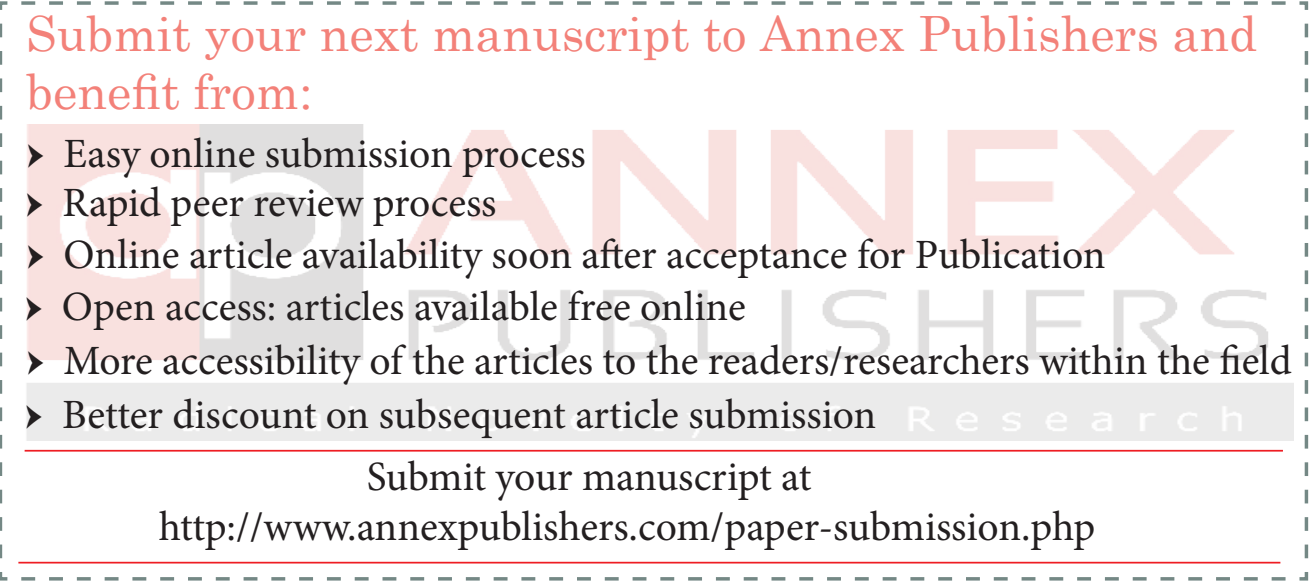

